\title{
Orbital cellulitis: review of 23 cases from Saudi Arabia
}

\author{
ERNEST HODGES AND KHALID F TABBARA \\ From the Department of Ophthalmology, College Of Medicine, King Saud University, and the Research \\ Department, King Khaled Eye Specialist Hospital, Riyadh, Saudi Arabia
}

SUMmARY We studied a total of 23 patients with orbital cellulitis and/or orbital abscess over a period of four years in Saudi Arabia. The study showed a high rate of abscess formation (12/23) and surgical intervention (17/23). Twelve out of 23 patients were 18 or more years of age. Furthermore, 12 of $23(52 \%)$ patients had blind eyes on admission and remained blind after treatment, and one patient died of cavernous sinus thrombosis. Only $7 / 23(30 \%)$ had a predisposing cause of primary sinus disease. This study of orbital cellulitis in a developing country presents a variation in disease pattern from previous reports and suggests that delay in the initiation of antibiotic therapy may lead to serious complications which may be life threatening. The course and outcome of orbital cellulitis may vary, depending on the predisposing factor, time of onset, associated systemic disease, or delay in initiation of treatment.

Orbital cellulitis is an ocular emergency that not only threatens vision but also can lead to life-threatening complications such as cavernous sinus thrombosis, meningitis, and brain abscess. ' In the management of orbital cellulitis physicians must recognise early signs and symptoms, be aware of predisposing conditions, and be prepared to administer prompt and appropriate therapy. Several recent reports ${ }^{2.13}$ from different countries document similar clinical disease patterns in infectious orbital disease. However, this report highlights the serious outcome when therapy is delayed and therefore underscores the differences in the course and prognosis of the disease between a developing country and countries with well established and widely available medical facilities.

\section{Materials and methods}

Patients. A total of 23 patients with a diagnosis of orbital cellulitis without abscess and orbital cellulitis with orbital or subperiosteal abscess were admitted to the King Khaled Eye Specialist Hospital (KKESH) during the period of January 1983 to December 1986. We studied retrospectively the charts of patients who were admitted to the hospital with acute infectious orbital disease.

Clinical and radiological examinations. In all cases the cardinal signs of orbital cellulitis, including

Correspondence to Dr Khalid F Tabbara, Research Department, King Khaled Eye Specialist Hospital. PO Box 7191. Riyadh 11462. Saudi Arabia. inflammatory proptosis or painful ophthalmoplegia, were present, indicating involvement behind the orbital septum. The diagnosis of orbital abscess was made when an abscess cavity was noted on computerised tomography scan or when pus was drained from the orbit or subperiosteal space at the time of surgery. All patients improved on the administration of appropriate antibiotics or surgical intervention. Radiological examinations were carried out in 20 of 23 patients and included CT scan in 16 of 23 patients and $x$-rays of the orbits and sinuses in 10 of 23 patients. One patient refused radiological examination, and two patients were not examined radiologically because they were known cases of endophthalmitis where the infection extended to the orbit.

Microbiological studies. Blood specimens and swabs from the periocular region and nasopharynx were obtained and cultured for bacteria and fungi. Specimens were cultured on blood agar (aerobic and anerobic conditions), chocolate agar, Sabouraud's agar, and thioglycolate. Occasionally material was cultured on Löwenstein-Jensen medium to rule out tuberculosis, and an antimicrobial removal device (ARD) was used if an organism was not identified and the patient had been on a previous regimen of antibiotic therapy.

Treatment. High dose intravenous broad spectrum antibiotics were given after cultures were obtained. If an orbital abscess cavity was noted on CT scan or if the clinical situation deteriorated while appropriate 
antibiotics were being administered, the patient was subjected to surgical drainage of the abscess. Patients with primary sinus disease were generally given 24 to 48 hours to respond to antibiotic therapy before the sinus was drained.

\section{Results}

CLINICAL AND RADIOLOGIC FINDINGS Of the 23 patients admitted for the treatment of orbital cellulitis or abscess $19(83 \%)$ were male and 4 $(17 \%)$ were female patients. The age range was 6 months to 66 years, with $12(52 \%)$ aged 18 years or more and $11(48 \%)$ under 18 . There were 13 cases of orbital cellulitis with abscess formation and 10 of orbital cellulitis without abscess.

From the onset of symptoms until hospital admission and initiation of medical treatment there was a delay ranging from 2 to 40 days (mean $7 \cdot 7$ days). Delay among patients with orbital cellulitis and abscess formation ranged from 2 to 40 days (mean 9.4 days) and among those with orbital cellulitis without abscess formation from 3 to 12 days (mean $5 \cdot 5$ days).

\section{PREDISPOSING FACTORS}

A predisposing cause could be determined in $20 / 23$ $(87 \%)$ patients. Of these, seven were secondary to primary sinus disease, five to penetrating injury, four to endophthalmitis, two to previous surgery, and one each to acute dacryocystitis and rhabdomyosarcoma complicated by sinusitis (Table 1). Two of the patients who had sinus disease were immunosuppressed: one was diabetic and the other was receiving systemic corticosteroids. The patient who received systemic steroids had previously undergone splenectomy and eventually developed cavernous sinus thrombosis, brain abscess, and died.

Of the seven patients with primary sinusitis five were between the ages of 9 to 25 , one was 48 , and one was a 60 year old. An additional patient with sinusitis complicating rhabdomyosarcoma was aged 8 . The ethmoid sinus was infected in all of the younger patients as well as in the 60 -year-old. The 48 -year-old

Table 1 Predisposing factors for orbital cellulitis

\begin{tabular}{lc}
\hline Factors & No. of cases \\
\hline Primary sinus disease & 7 \\
Penetrating injury & 5 \\
Endophthalmitis & 4 \\
Previous surgery & 2 \\
Dacryocystitis & 1 \\
Rhabdomyosarcoma & 1 \\
Undetermined & 3 \\
Total & 23 \\
\hline
\end{tabular}

was the only patient who had a primary focus of infection in the maxillary sinus. One patient with diabetes mellitus developed phycomycosis.

Four patients had been injured in motor vehicle accidents (aged 2, 5, 7, and 21 years) and a 4-year-old girl has suffered a penetrating injury with a knife.

Endophthalmitis caused orbital cellulitis as a result of a corneal ulcer in four patients. Two of the four had ulcers that were complications of previous surgical procedures. These four patients ranged in age from 40 to 66 years.

MICROBIOLOGICAL RESULTS

Causative organisms could be identified in $17 / 23$ (74\%) of the patients. Positive cultures were obtained from an abscess site in the orbit or from an infected cornea or sinus. No positive blood cultures were obtained.

The organisms isolated included Staphylococcus aureus (8), Streptococcus pneumoniae (5), $\alpha$-haemolytic streptococci (not Streptococcus pneumoniae) (3), and Staphylococcus epidermidis (1), Haemophilus influenzae (1), anaerobic streptococci (1), and a fungus of class Phycomycetes (1) (Table 2). Three of the patients had mixed infections, two of which followed trauma. Four patients had endophthalmitis leading to orbital cellulitis and this was caused by Streptococcus pneumoniae in two patients, Staphylococcus aureus in one patient, and Haemophilus influenzae in one. No patients had an anaerobic agent as the sole cause. Only one patient had fungal cellulitis, a 60 -year-old poorly controlled diabetic, with an ethmoid sinus infection.

\section{RADIOLOGICAL FINDINGS}

Computerised tomography scans of the orbits and sinuses were obtained in 16 of 23 patients; the correlation between them and the clinical and surgical course was good. In two cases, however, an abscess cavity could not be diagnosed radiologically but was confirmed at surgery. Plain views of the orbit were taken in many of the patients who had a CT scan

Table 2 Causative agents* in orbital cellulitis

\begin{tabular}{ll}
\hline Agent & No. of patients \\
\hline Staphylococcus aureus & 8 \\
Streptococcus pneumoniae & 5 \\
a-haemolytic streptococci (not Streptococcus & \\
$\quad$ pneumoniae) & 3 \\
Staphylococcus epidermidis & 1 \\
Haemophilus influenzae & 1 \\
Anaerobic streptococci & 1 \\
Phycomycetes & 1 \\
Not cultured & 6 \\
\hline
\end{tabular}

*Three patients had mixed infections. 
Table 3 Surgery performed for orbital infections

\begin{tabular}{ll}
\hline Surgery & No. of procedures \\
\hline $\begin{array}{l}\text { Drainage of orbital abscess } \\
\text { Drainage of sinus (ethmoid 6, maxillary 6, } \\
\quad \text { frontal 1) }\end{array}$ & 10 \\
Evisceration & 4 \\
Removal of infected supramid plate and glue & 1 \\
Total number of procedures performed on 17 & 22 \\
patients & \\
\hline
\end{tabular}

and also in $4 / 7$ of the remaining patients, but in no instances were conventional radiographs of the orbit helpful in delineating abscess formation.

\section{OUTCOME}

All the patients were treated with intravenous antibiotics effective for both Gram-negative and Grampositive organisms. Seventeen were subjected to surgery because of an orbital infection. Several of these had more than one operation either at one sitting or staged over the treatment period. The surgical interventions included drainage of an orbital abscess in 10 patients, drainage of a sinus or sinuses in seven, evisceration in four, and removal of an infected supramid plate and glue in one (Table 3 ). Twelve of 23 patients had blind eyes on admission and remained blind after treatment.

Apart from the decrease in vision and the surgical operations mentioned the following complications were noted in eight patients: one had an orbital haematoma postoperatively, two developed infected orbital plates with a fistula to the skin, two had recurrent orbital cellulitis due to undertreatment with antibiotics, two developed exposure keratitis, one extruded the acrylic spherical implant, and one developed large-angle exotropia after drainage of an abscess. In one patient there was spontaneous rupture of an abscess through the lid, and septic cavernous sinus thrombosis was observed in another, resulting in death. A progressive increase was noted in the number of complications, depending on how long the patient waited for admission and treatment after the symptoms or predisposing factors began (Fig. 1).

\section{Discussion}

The fact that only 23 patients in four years were identified with the diagnosis of orbital cellulitis with or without abscess formation suggests that many patients may be treated elsewhere. Some authors ${ }^{2}$ require the presence of bacteria in the postseptal tissues for diagnosis, while others" require only inflammatory cells (sterile cellulitis) for diagnosis, with the bacteria being contained in the sinuses, globe, or lacrimal sac. Clearly it is often difficult to substantiate the actual penetration of bacteria into the soft tissues of the orbital cavity.

When a patient presents with infectious orbital disease, one may quickly conclude that orbital cellulitis is present because this is more frequently encountered than orbital abscess. ${ }^{3-10}$ The incidence of abscess formation in orbital cellulitis varies from 0 to $25 \%$. $^{3-111}$ In our series $13 / 23(57 \%)$ developed orbital abscess; this is certainly greater than that reported in previous series and reflects a delayed diagnosis or treatment. In view of the fact that KKESH is a tertiary care facility, this also may indicate the bias in this highly selected sample of patients and may not reflect the true incidence of orbital abscess formation among patients with orbital cellulitis.

Because of the high incidence of abscess formation in this situation, management must be very aggressive. Management should include (1) obtaining adequate specimens for microbiological studies, (2) delivering high-dose intravenous antibiotic therapy immediately, (3) altering antibiotics according to sensitivities, and (4) intervening surgically at the proper time. If no organisms are seen by Gram stain, a $\beta$-lactamase resistant agent should be started until sensitivities are obtained. If the patient is less than 5 years of age, ampicillin or chloramphenicol should be added to cover for Haemophilus. In patients with sinus disease 24 to 48 hours are usually allowed for a response to antibiotic therapy to occur before

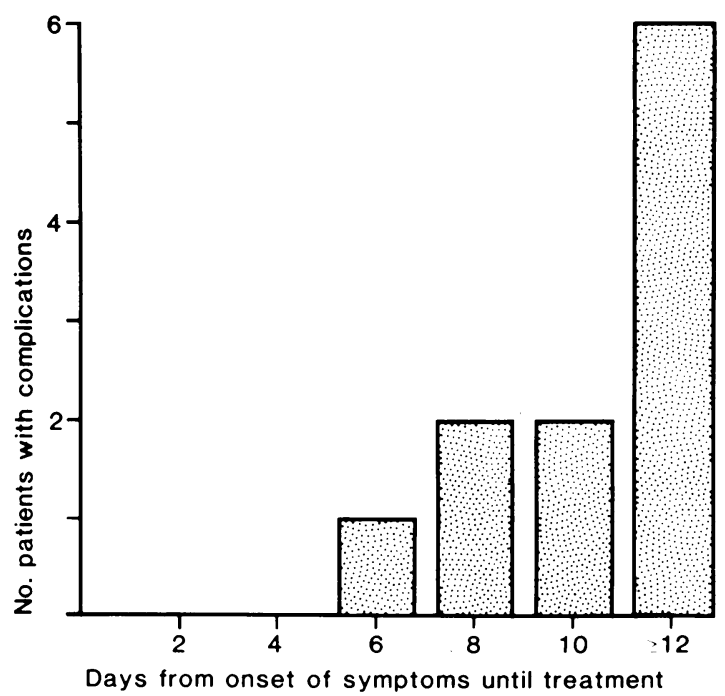

3 patients had more than one complication

Fig. 1 Complications related to delay in treatment in orbital cellulitis. 
surgical drainage of the sinus(es). An abscess cavity is considered a surgical emergency and should be drained promptly.

Because of the high rate of abscess formation the number of patients undergoing surgery was also very large $(73 \%)$. Previous studies ${ }^{+4}$ indicate a range of 0 to $38 \%$ of patients requiring surgery. Of the 13 patients in our study who developed orbital abscess 12 had surgery to drain the abscess (occasionally in conjunction with sinus drainage), and one orbital abscess spontaneously ruptured through the lid.

The patient who died from cavernous sinus thrombosis had previous splenectomy following a motor vehicle accident and was maintained on steroid therapy.

Few studies give an accurate age delineation of orbital cellulitis because most reports are from institutions that treat only children. ${ }^{i-57-9}$

The CT scan was helpful in delineating the amount of involvement of disease process and indication for surgery. But in no cases were conventional radiographs of the orbit useful in delineating orbital abscess formation. In two cases, however, a CT scan could not define an orbital abscess suspected clinically and proved by surgical intervention. Krohel et al. have noted that an abscess developing over 24 to 38 hours may produce only non-specific inflammatory signs on CT scan and not identify an abscess. ${ }^{14}$

This report draws attention to several differences in the presentation of orbital cellulitis and abscess formation in Saudi Arabia as compared with previously reported studies. When confronted with patients in developing countries, one must bear in mind that the presentation of a disease may vary considerably from what has been reported previously.

\section{References}

1 Jones DB. Microbial preseptal and orbital cellulitis. In: Duane TD, ed. Clinical ophthalmology. New York: Harper and Row. 1976; 4 : chapter 25.

2 Chandler JR, Langenbrunner DJ, Stevens ER. The pathogenesis of orbital complications in acute sinusitis. Laryngoscope 1970; 80: 1414-28.

3 Gellady AM, Shulman ST, Ayoub EM. Periorbital and orbital cellulitis in children. Pediatrics 1978; 61: 272-7.

4 Noel LP. Clarke WN, Peacocke TA. Periorbital and orbital cellulitis in children. (an J Ophthalmol 1981: 16: 178-80.

5 Fearon B, Edmonds B, Bird R. Orbital-facial complications of sinusitis in children. Laryngoscope 1979: 89: 947-53.

6 Bergin DJ. Wright JE. Orbital cellulitis. Br J Ophthalmol 1986: 70: $174-8$.

7 Schramm VL, Myers EN, Kennerdell JS. Orbital complications of acute sinusitis: evaluation, management, and outcome. Otolaryngol Head Neck Surg 1978: 86: 221-30.

8 Weiss A. Friendly D, Eglin K, Chang M, Gold B. Bacterial periorbital and orbital cellulitis in childhood. Ophthalmology 1983: 90: 195-203.

9 Watters EC. Wallar PH, Hiles DA, Michaels RH. Acute orbital cellulitis. Arch Ophthalmol 1976: 94: 785-8.

10 Hornblass A. Herschorn BJ, Stern K, Grimes C. Orbital abscess. Surv Ophthalmol 1984: 29 (3): 169-78

11 Jarrett WH, Gutman EA. Ocular complications of infection in the paranasal sinuses. Arch Ophthalmol 1969; 81: 683-8.

12 Krohel GB, Krauss HR, Winnick J. Orbital abscess. Presentation, diagnosis, therapy, and sequelae. Ophthalmology 1982: 89: $492-8$.

1.3 Harris (jJ. Subperiosteal abscess of the orbit. Arch ()phthalmol 1983: 101: 751-7.

14 Krohel GB, Krauss HR, Christensen RE, Minckler D. Orbital abscess. Arch Ophthalmol 1980; 98: 274-6.

Accepted for publication 3 March 1988. 